\title{
Inhibitory effects of STAT3 decoy oligodeoxynucleotides on human epithelial ovarian cancer cell growth in vivo
}

\author{
XIAOLEI ZHANG ${ }^{1}$, PEISHU LIU ${ }^{1}$, BO ZHANG $^{2}$, HONGLUAN MAO $^{1}$, LIANG SHEN ${ }^{1}$ and YANHUI MA ${ }^{1}$ \\ ${ }^{1}$ Department of Obstetrics and Gynecology, Qilu Hospital of Shandong University, Jinan, Shandong 250012; \\ ${ }^{2}$ Department of Clinical Medicine, The Second Hospital of Shandong University, Jinan, Shandong 250033, P.R. China
}

Received March 30, 2013; Accepted June 13, 2013

DOI: $10.3892 / \mathrm{ijmm} .2013 .1431$

\begin{abstract}
The signal transducer and activator of transcription 3 (STAT3) regulates target gene expression by binding to a consensus DNA sequence within the promoter of the target genes. The constitutive activation of STAT3 has been shown to contribute to tumorigenesis in ovarian cancer and it has been reported to be a key factor for drug resistance in ovarian cancer. STAT3-specific decoy oligodeoxynucleotides (ODNs) (STAT3 decoy ODNs) that contain a consensus DNA sequence inhibit the transcriptional activity of STAT3, leading to cancer cell death. However, their mechanisms of action are unclear and little information is available as to the effects and the toxicity of STAT3 decoy ODNs in vivo. In this study, we established subcutaneous xenografts of SKOV3 human ovarian cancer cells in nude mice, evaluated the antitumor effects of STAT3 decoy ODNs on xenografted nude mice, and investigated the mechanisms behind the antitumor effects of STAT3 decoy ODNs targeting the STAT3 signaling pathway in vivo. The results revealed that the STAT3 decoy ODN inhibited ovarian cancer cell growth and promoted ovarian cancer cell apoptosis in vivo. Western blot analysis indicated that the STAT3 decoy ODN downregulated the protein expression levels of matrix metalloproteinase (MMP)-2, MMP-9 and Bcl-2, and upregulated the protein expression levels of caspase-3 in vivo. $\mathrm{H} \& \mathrm{E}$ staining was used to detect the side-effects of the STAT3 decoy ODN in the vital organs of the nude mice. We found that there were no significant abnormalities in the vital organs of the nude mice apart from slight inflammation and necrosis in parts of the hepatic lobule. The data from the present study suggest that decoy ODNs targeting STAT3 may be an effective therapeutic approach for the treatment of ovarian cancer in vivo.
\end{abstract}

Correspondence to: Dr Xiaolei Zhang or Dr Peishu Liu, Department of Obstetrics and Gynecology, Qilu Hospital of Shandong University, 107 Wen-hua Xi Road, Jinan, Shandong 250012, P.R. China

E-mail: doctor_zxl@126.com

E-mail: dr_peishuliu@126.com

Key words: ovarian cancer, signal transducer and activator of transcription 3, decoy oligodeoxynucleotides, in vivo

\section{Introduction}

Epithelial ovarian cancer (EOC) is described as a silent killer as patients always present with local invasion or distant metastasis at first diagnosis. In the developed world, ovarian cancer is the leading cause of gynecological cancer-related mortality among women (1). Surgical cytoreduction and treatment with chemotherapeutic agents, such as cisplatin and paclitaxel (Taxol), are commonly used to treat this malignancy. However, the majority of ovarian cancer survivors eventually suffer from recurrent disease that develops resistance to multiple chemotherapeutic agents; thus, EOC is a disease with a high mortality rate. Long-term survival is achieved in less than a third of patients with advanced-stage ovarian cancer (2).

The Janus kinase/signal transducer and activator of transcription (JAK/STAT) signaling pathway is an important pathway by which cytokines transfer information from the surface of the cell into the nucleus. STAT3 is a crucial member of the JAK/STAT signaling pathway. STAT3 has been described as a key regulator of cell survival and proliferation (3). It is constitutively activated in a variety of tumor cell lines and primary tumors, including prostate, breast, head and neck cancer, multiple myeloma and glioma (4-7). STAT3 is activated by various protein tyrosine kinases, JAK and the proto-oncogene tyrosine-protein kinase (Src), as well as membrane-bound growth factor receptor tyrosine kinases, such as the epidermal growth factor receptor (EGFR) $(8,9)$. Thus, the functions of activated STAT3 proteins vary, and include cell growth, differentiation, development, apoptosis and angiogenesis $(10,11)$. The constitutive activation of STAT3 has been shown to contribute to tumorigenesis in ovarian cancer (12). STAT3 has also been reported to be a key factor for drug-resistance in ovarian cancer (13). Therefore, STAT3 may be a potential molecular target for the treatment of cancer (14).

The constitutive activation of STAT3 in tumor cells points to STAT3 as a valuable target for attacking tumor cells. Moreover, STAT3 is not essential for the functioning of mature cells (15). Thus, it is a highly valuable target for inducing tumor cell death; however, STAT3 lacks more specific inhibitors. Several strategies have been investigated to target the signaling pathway of STAT3, including RNA interference (using siRNA), dominant-negative, antisense and 'decoy' oligodeoxynucleotides (ODN) technology (16-18). A recent study reported that a STAT3 decoy oligonucleotide induced cell death in a human 
colorectal carcinoma cell line by blocking nuclear transfer (19). In our previous study, we utilized the decoy ODN technology to examine the effects of a STAT3 decoy ODN on human ovarian cancer cells in vitro and found that the STAT3 decoy ODN decreased the invasive capability of the cancer cells and enhanced the sensitivity of ovarian cancer cells to paclitaxel (20). However, there is little information available as to the effects of STAT3 decoy ODNs on cancer in vivo, and little information as to its toxicity in vivo.

In this study, we established subcutaneous xenografts of SKOV3 human ovarian cancer cells in nude mice, examined the antitumor effects of STAT3 decoy ODNs on xenografted nude mice, investigated the potential mechanisms of the STAT3 decoy ODNs, and examined the side-effects of the STAT3 decoy ODNs on the vital organs of nude mice.

\section{Materials and methods}

Cell line and cell culture. The SKOV3 human ovarian epithelial cancer cell line was provided by the Qilu Hospital Biotechnology Center, Shandong University, Jinan, China. The cells were cultured in RPMI-1640 medium (Gibco-BRL, Grand Island, NY, USA) supplemented with $10 \%$ heat-inactivated fetal bovine serum (FBS; Gibco-BRL) and incubated under standardized conditions $\left(37^{\circ} \mathrm{C}, 5 \%\right.$ carbon dioxide).

STAT3 decoy and scrambled ODN. Phosphorothioate sense and antisense strands of STAT3 decoy or scrambled control ODNs were synthesized using the Expedite ${ }^{\mathrm{TM}}$ Nucleic Acid Synthesis System (Sangon Biotechnology, Shanghai, China). The STAT3 decoy ODN sequence was 5'-CATTTCCCGTAA ATC-3' and 3'-GTAAAGGGCATTTAG-5' and the scrambled ODN sequence was 5'-CATCTTGCCAATATC-3' and 3'-GTAGAACGGTTA TAG-5' $(21,22)$. The sense and antisense strands were then prepared by annealing complementary single-stranded ODNs by heating to $95^{\circ} \mathrm{C}$ for 10 min followed by cooling to room temperature slowly over a period of $2 \mathrm{~h}$.

Subcutaneous xenografts in nude mice. Female nude mice (BALB/c, 4-5 weeks old) were purchased from the Shanghai Experimental Animal Center (Chinese Academy of Sciences, Shanghai, China). The mice were housed and maintained under specific pathogen-free conditions according to the experimental animal guidelines and approved by the Institutional Animal Care and Use Committee of Shandong University. The tumor model was established according to a previous study (23). SKOV3 cells were harvested and resuspended in RPMI-1640 medium. A total of $200 \mu 1$ SKOV3 cells $\left(2 \times 10^{7} / \mathrm{ml}\right)$ were injected into the right flanks of the mice. Two weeks later, the mice were randomly assigned to 3 groups with 5 mice in each group (STAT3 decoy ODN treatment group, STAT3 scrambled ODN treatment control group and PBS treatment control group). ODN (50 $\mu \mathrm{g}$ in $50 \mu \mathrm{l}$ PBS) was intratumorally injected every other day for 30 days. Tumor sizes were measured by length (l) and width (w) every 4 days, and the tumor volumes were calculated according to the following formula: tumor volume $=1 \mathrm{w}^{2} / 2$. The mental state, diet and stool of the mice were observed daily. Twelve hours after the final injection, the mice were sacrificed. The tumors, heart, liver and kidneys were removed, parts of them were fixed in formalin and embedded in paraffin, and parts of them were frozen at $-80^{\circ} \mathrm{C}$ for western blot analysis. Paraffin sections were made from the tumor, heart, liver and kidney tissues of the nude mice, and the tissues were stained with hematoxylin and eosin (H\&E).

TUNEL assay for the detection of apoptosis induced by STAT3 decoy ODN in vivo. To detect the poptotic cells in the tumor tissues, TUNEL assay, using a Fluorometric TUNEL System (KeyGen Biotech, Nanjing, China) was performed according to the manufacturer's instructions. The tissue sections were rinsed with PBS for 5 min following incubation with proteinase $\mathrm{K}(18 \mu \mathrm{g} / \mathrm{ml})$ for $20 \mathrm{~min}$, and then blocked with fetal bovine serum for $15 \mathrm{~min}$ at room temperature. TdT reaction mix $(50 \mu \mathrm{l})$ was added to the sections followed by incubation in a humidified chamber for $60 \mathrm{~min}$ at $37^{\circ} \mathrm{C}$. The the sections were then rinsed with PBS and observed under a fluorescence microscope. Cell nuclei with green fluorescent staining were defined as TUNEL-positive nuclei. To quantify the TUNEL-positive cells, the number of green fluorescencepositive cells was counted in 5 random fields of view on each section at X200 magnification.

Western blot analysis. The tumor tissues were lysed in lysis buffer. Sodium dodecyl sulfate-polyacrylamide gel electrophoresis (SDS-PAGE) was performed as previously described (24). The whole cell extracts (30 $\mu \mathrm{g} /$ lane) were separated by SDS-PAGE and transferred onto nitrocellulose membranes (Millipore, Bedford, MA, USA). The membranes were blocked in Tris-buffered saline with 5\% (w/v) non-fat dry milk, and then incubated with a primary antibody against $\beta$-actin, MMP-2, MMP-9, Bcl-2 and caspase-3 (1:1,000; Santa Cruz Biotechnology, Inc., Santa Cruz, CA, USA) at $4^{\circ} \mathrm{C}$. After washing with TBST 3 times, the membranes were incubated with horseradish peroxidase-conjugated secondary antibody. Immunoreactive proteins were visualized using an enhanced chemiluminescence (ECL) detection system (Pierce, Rockford, IL, USA). The bands were examined using a densitometer analysis system (Flurochem 9900-50; Alpha Innotech, San Leandro, CA, USA). Band density was analyzed using BandScan software (Glyko, Novato, CA, USA) and the results were expressed as a ratio of the protein of interest/ $\beta$-actin to correct for loading for each sample.

Statistical analysis. All statistical analyses were performed using SPSS 17.0 software. All data are expressed as the means \pm SD of at least 3 independent experiments. The differences between groups were analyzed using the Student's t-test; P-values $<0.05$ were considered to indicate statistically significant differences in all cases.

\section{Results}

STAT3 decoy ODN inhibits ovarian cancer cell growth in vivo. The SKOV3 cells formed xenografts in the nude mice, and the average time taken for the tumors to be formed was 10-12 days. Images of the xenografted nude mice are shown in Fig. 1. Tumor growth curves showed that the growth of the tumors treated with the STAT3 decoy ODN was significantly inhibited compared with the other treatment groups (Fig. 2). 


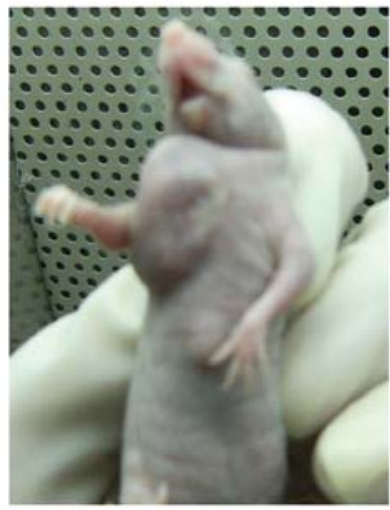

PBS

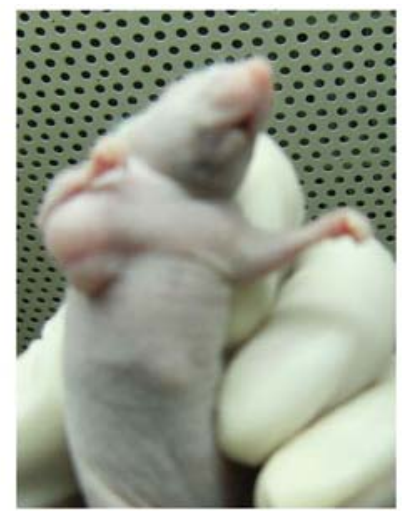

Scrambled

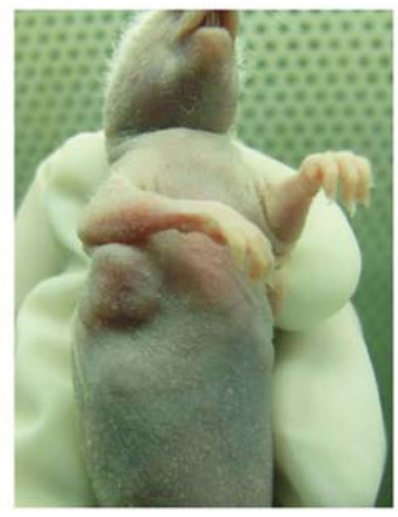

decoy

Figure 1. Images of mouse xenografts. Mice were subcutaneously injected with SKOV3 human ovarian cancer cells and then treated with either the STAT3 decoy ODN, the STAT3 scrambled ODN, or PBS.

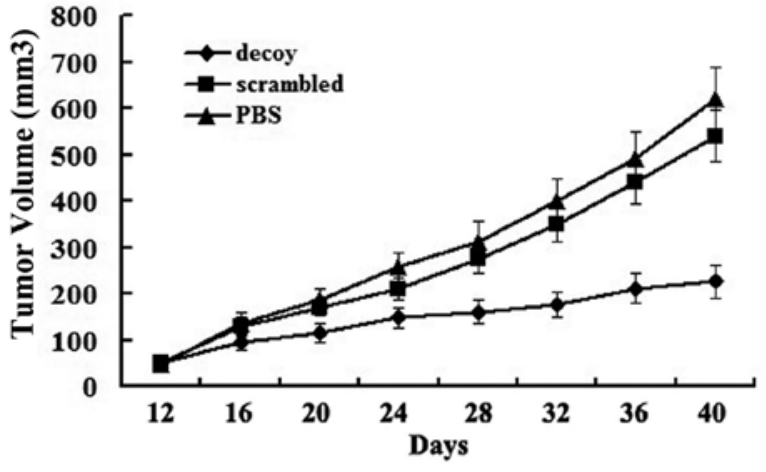

Figure 2. Tumor growth curves showing that the STAT3 decoy ODN significantly inhibited tumor growth compared with the PBS and STAT3 scrambled treatment groups.

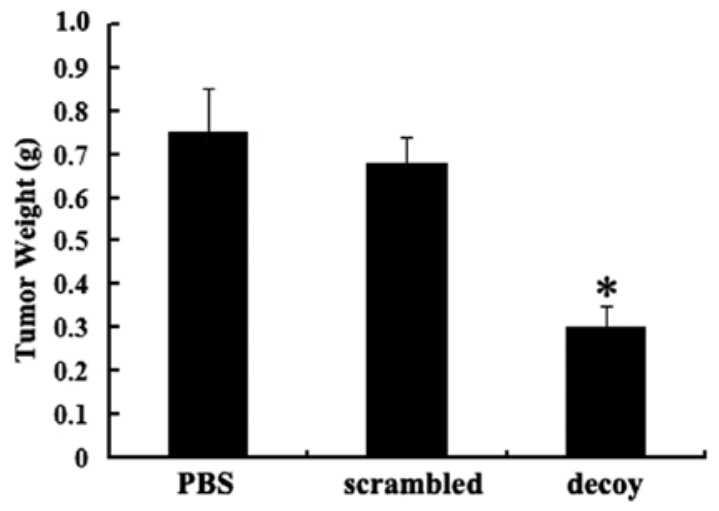

Figure 3. Average weights of tumor tissues in the 3 treatment groups. The average weight of the tumor tissues in the STAT3 decoy ODN treatment group was significantly lower than that in the other 2 control groups $\left({ }^{*} \mathrm{P}<0.05\right)$.
After the treatment was terminated, the average volume of the xenografts was as follows: $620 \pm 68 \mathrm{~mm}^{3}$ in the PBS group, $540 \pm 55 \mathrm{~mm}^{3}$ in the scrambled group and $226 \pm 35 \mathrm{~mm}^{3}$ in the decoy group. The average volume of the xenografts in the decoy group decreased significantly compared with the PBS and scrambled group $(\mathrm{P}<0.05)$. However, the difference between the PBS and scrambled groups was not statistically significant. The average weight of the tumor tissues in the decoy group was significantly lower than that in the other control groups $(\mathrm{P}<0.05)$ (Fig. 3).

STAT3 decoy ODN promotes ovarian cancer cell apoptosis in vivo. A fluorometric TUNEL assay was performed to detect the apoptotic cells in the tumor tissues of the nude mice. Cell nuclei with green fluorescent staining were defined as apoptotic cells. TUNEL assay revealed that there were $43 \pm 7$ apoptotic cells/high power field (/HPF) in the group treated with the STAT3 decoy ODN, while there were $11 \pm 3$ apoptotic cells in the scrambled group and $13 \pm 4$ apoptotic cells in the PBS group (Fig. 4). The difference between the decoy group and the other 2 groups was statistically significant $(\mathrm{P}<0.05)$. These results suggested that the STAT3 decoy ODN promoted ovarian cancer cell apoptosis in vivo.
Side-effects of STAT3 decoy ODN on tumor tissues and vital organs of nude mice. In the tumor tissue sections, $\mathrm{H} \& \mathrm{E}$ staining revealed karyomegaly, anachromasis and karyokinesis in the cancer cells (Fig. 5). Necrosis was observed partly in the xenograft tissues. The sections of the heart and kidney tissues showed no significant abnormalities. However, 1 in 5 nude mice treated with the STAT3 decoy ODN had slight inflammation and necrosis in parts of the hepatic lobule.

Effects of STAT3 decoy ODN on the protein expression of MMP-2, MMP-9, Bcl-2 and caspase-3 in vivo. Western blot analysis was used to examine the protein expression of MMP-2, MMP-9, Bcl-2 and caspase-3 in the xenograft tissues of the nude mice. Compared with the PBS and scrambled treatment group, the protein expression level of MMP-2, MMP-9, Bcl-2 in the STAT3 decoy ODN treatment group was significantly downregulated, while the protein expression level of caspase-3 was significantly upregulated (Fig. 6).

\section{Discussion}

STAT3 has been recognized as an oncogene, due to its oncogenic role (25). Constitutive STAT3 activation is frequently involved 


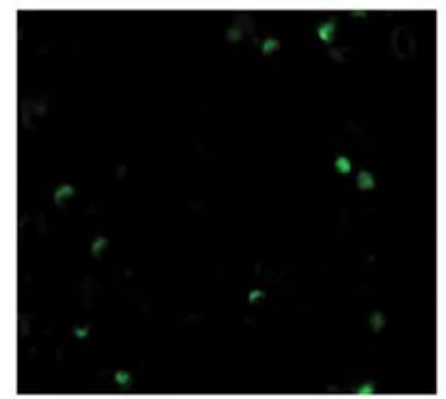

PBS

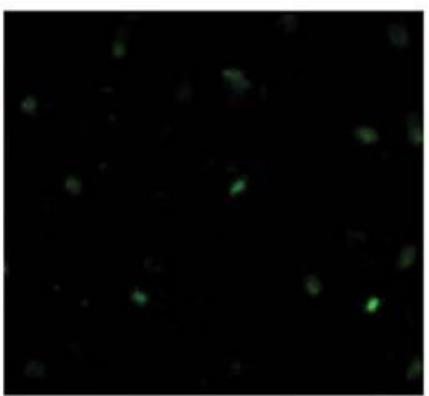

scrambled

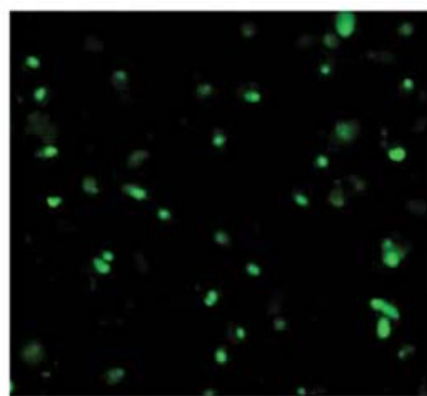

decoy

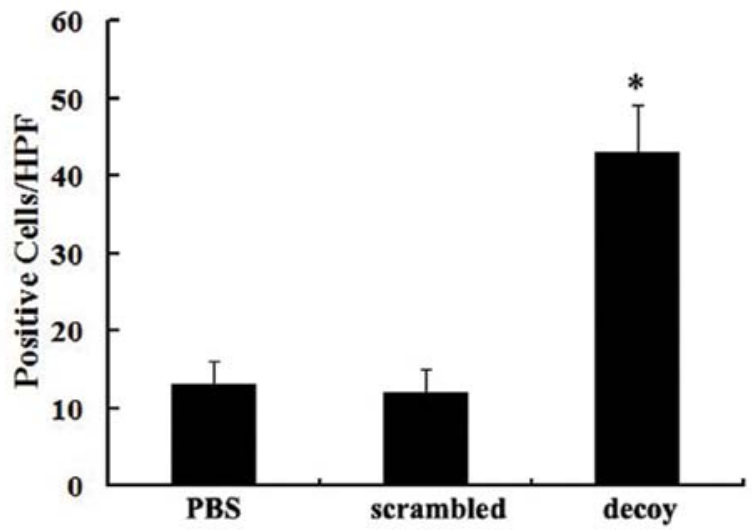

Figure 4. TUNEL assay revealed that the STAT3 decoy ODN promoted ovarian cancer cell apoptosis in vivo. Cell nuclei with green fluorescent staining were defined as apoptotic cells. The histogram shows that the number of apoptotic cells in the STAT3 decoy ODN treatment group was increased compared with the other 2 control groups $\left({ }^{*} \mathrm{P}<0.05\right)$.
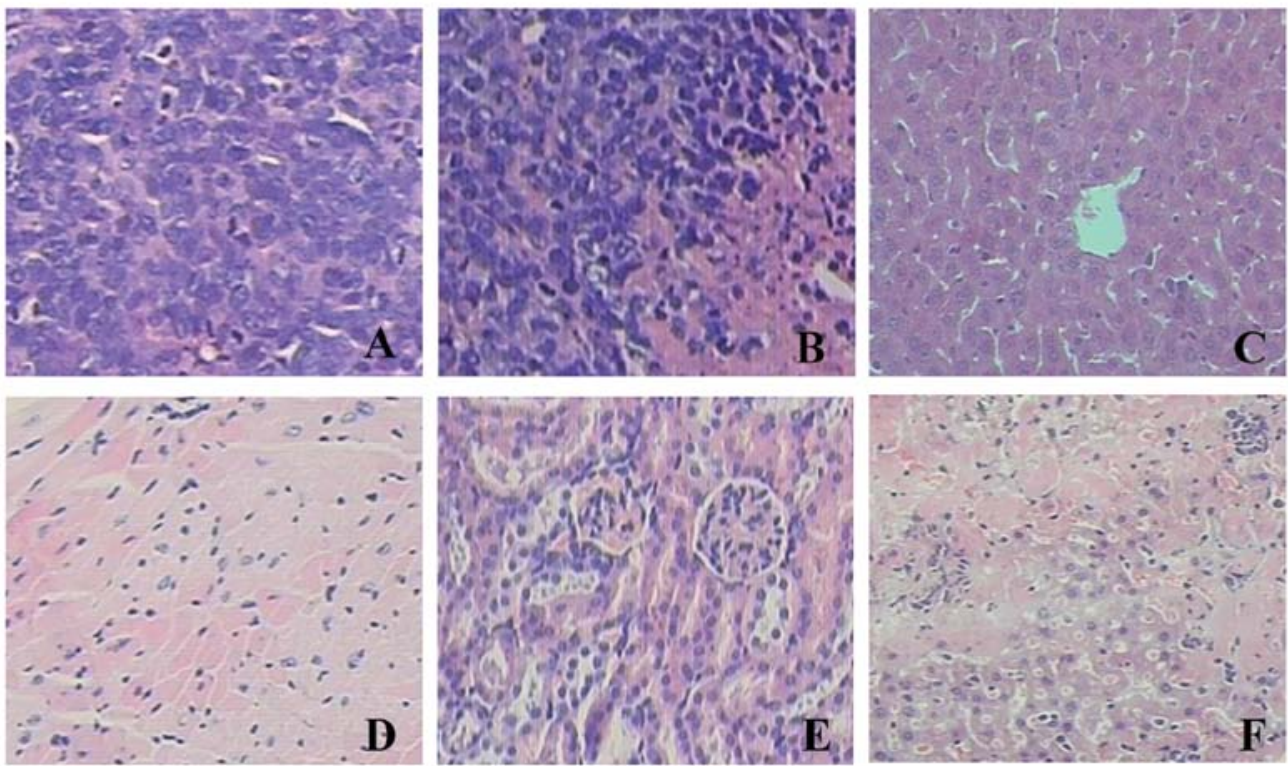

Figure 5. H\&E staining of xenograft tumor tissues of the heart, kidneys and liver in nude mice. (A) Karyomegaly, anachromasis and karyokinesis can be seen in the xenograft tumor cells. (B) Necrosis can partly be seen in the xenograft tumor tissues. (C) The liver tissue sections are relatively normal in the nude mice treated with the STAT3 decoy ODN, apart from (F) slight inflammation and necrosis in parts of the hepatic lobule. (D and E) sections of heart and liver tissues showed no significant abnormalities.

in uncontrolled tumor cell proliferation and therefore constitutes a valuable target for antitumor therapy (26). Studies have shown that elevated levels of total and/or tyrosine phosphorylated STAT3 (pSTAT3) in the tumor are associated with decreased survival rates in cancer patients, and this suggests that STAT3 may serve as a therapeutic target (27). A previous study reported that STAT3 is associated with the aggressive biological behavior of ovarian cancer cells (28). However, the specific mechanisms involved are unclear. Decoy ODNs have been shown to efficiently induce cell death in a variety of cellular systems (29) and to have potential for the specific targeting of tumor cells. The decoy ODN technology is a novel tool, which has advantages of 


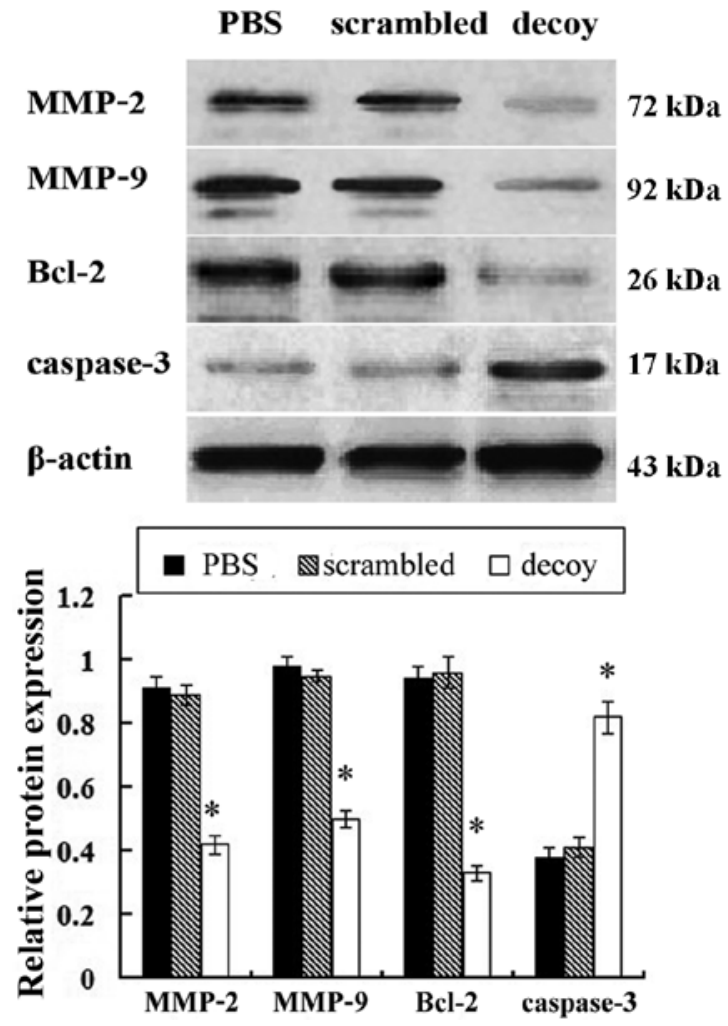

Figure 6. Effects of STAT3 decoy ODN on the protein expression of matrix metalloproteinase (MMP)-2, MMP-9, Bcl-2 and caspase-3 in vivo. Compared with the PBS and scrambled treatment groups, the protein expression level of MMP-2, MMP-9, Bcl-2 in the STAT3 decoy ODN treatment group was significantly decreased, while the protein expression level of caspase- 3 was significantly increased.

low cost, specificity, simplicity and effectiveness. Moreover, the sequence of decoy ODNs is relatively stable. This technology has been successfully used to inhibit STAT3 pathway activation in squamous cell carcinoma of the head and neck (SCCHN), pancreatic cancer and human lung cancer. However, studies on the effects of STAT3 decoy ODNs on ovarian cancer are limited, and the biological effects of STAT3 decoy ODNs in vivo have not yet been fully elucidated.

In this study, to elucidate the antitumor effects of STAT3 on ovarian cancer in vivo, we used the novel technology, decoy ODNs, to regulate the STAT3 pathway in xenografted nude mice. The major findings of the present study were as follows: i) STAT3 decoy ODNs inhibited ovarian cancer cell growth and promoted ovarian cancer cell apoptosis in vivo; ii) STAT3 decoy ODNs downregulated the protein expression levels of MMP-2, MMP-9, Bcl-2 and upregulated the protein expression level of caspase-3 in vivo; iii) STAT3 decoy ODNs may have side-effects on the livers of nude mice.

We monitored tumor growth during the treatment period, and used tumor growth curves to reflect the inhibitory effects of 3 different treatments. The growth curves revealed that the growth of the tumors treated with STAT3 decoy ODN was significantly inhibited compared with the other treatment groups (Fig. 2). After the treatment was terminated, we removed the tumor tissues from the nude mice. The average volume and average weight of the tumor tissues in the decoy group were significantly lower than those in the other control groups $(\mathrm{P}<0.05)$. These findings indicated that the STAT3 decoy ODN inhibited ovarian cancer growth in the nude mice. Studies have shown that STAT3 decoy ODNs induce cancer cell apoptosis in vitro $(20,21)$. In this study, in order to detect the apoptotic cells in the tumor tissues from the nude mice, fluorometric TUNEL assay was performed. The results revealed that the number of apoptotic cells/HPF in the STAT3 decoy ODN treatment group was higher than that in the PBS and scrambled groups. These results suggested that the STAT3 decoy ODN promoted ovarian cancer cell apoptosis in vivo. These results are consistent with those of other studies, showing that blocking the activation of STAT3 suppresses tumor growth and induces tumor cell apoptosis in vivo $(22,30)$.

In this study, we used western blot analysis to detect the expression levels of apoptosis-related proteins. The protein expression levels of MMP-2, MMP-9, Bcl-2 in the STAT3 decoy ODN treatment group significantly decreased, while the protein expression level of caspase-3 significantly increased, compared with the PBS and scrambled treatment group. MMPs are zincdependent endopeptidases able to degrade components of the basement membrane and the extracellular matrix (ECM) (31). MMP-2 and MMP-9 are believed to be vital in the invasion of malignant tumors and angiogenesis. A previous study found that MMP-2 and MMP-9 induced the release of vascular endothelial growth factor (VEGF) in ovarian carcinoma cells, and promoted ascite formation (32). Our results demonstrated that the intratumoral injection of STAT3 decoy ODN decreased the expression of MMP-2 and MMP-9 in the tumor tissues of nude mice. This finding suggests that the downregulation of MMP-2 and MMP-9 may be one of the mechanisms involved in the STAT3 decoy ODN inhibition of cancer cell invasion and metastasis. Bcl-2 is a type of anti-apoptotic protein, the tumorigenic potential of which has been demonstrated in animal models and in certain human tumors (33). The anti-apoptotic protein, $\mathrm{Bcl}-2$, is associated with the inhibition of apoptosis, and its expression correlates with chemoresistance in cancer patients. Several studies have demonstrated that the inhibition of STAT3 by flavonoids and synthetic compounds results in tumor cell apoptosis in vitro $(34,35)$. In our study, we found that cancer cell apoptosis was promoted and that the expression of Bcl-2 was decreased following the treatment of mouse xenografts with STAT3 decoy ODN. This result indicates that STAT3 decoy ODNs promote cancer cell apoptosis by inhibiting the anti-apoptotic protein, $\mathrm{Bcl}-2$, in vivo. Caspase-3 is a central effector caspase in a number of cell types, leading to DNase activation followed by DNA fragmentation (36). Activated caspase- 3 has been shown to translocate to the nucleus during apoptosis and to play an important role in the nuclear changes in apoptotic cells (37). Moreover, our study showed that the expression of caspase- 3 was downregulated following treatment with STAT3 decoy ODNs.

Studies on the toxicity of STAT3 decoy ODNs are limited. In this study, we detected the biological effects of STAT3 decoy ODNs on heart, liver and kidney tissues from xenografted nude mice. In the sections of xenograft tissues (stained with H\&E), we observed karyomegaly, anachromasis and karyokinesis in the cancer cells. Necrosis was observed partly in the xenograft tissues. The sections of the heart and liver tissues showed no significant abnormalities. However, 1 in 5 nude mice treated with STAT3 decoy ODN showed some abnormalities in the organs, such as slight inflammation and necrosis in parts of 
the hepatic lobule. These findings suggest that STAT3 decoy ODNs may have side-effects on the livers of nude mice. The biological effects of STAT3 decoy ODNs on tissues of the heart, liver and kidneys in vivo require further investigation.

In conclusion, our study provides evidence that STAT3 decoy ODNs suppress growth and induce the apoptosis of ovarian cancer cells in vivo. The molecular mechanisms behind the inhibitory effects of STAT3 decoy ODNs may involve the downregulation of the protein expression of MMP-2, MMP-9 and $\mathrm{Bcl}-2$ and the upregulation of the protein expression of caspase-3 in vivo. Moreover, we examined the side-effects of STAT3 decoy ODNs on the vital organs of nude mice. We found that there were no significant abnormalities in the vital organs of the nude mice apart from slight inflammation and necrosis in parts of the hepatic lobule. Taken together, the data presented in this study demonstrate that the blockade of aberrantly activated STAT3 with decoy ODNs may be an efficient strategy for the treatment of ovarian cancer in vivo.

\section{References}

1. Jemal A, Siegel R, Ward E, Murray T, Xu J, Smigal C and Thun MJ: Cancer statistics. CA Cancer J Clin 56: 106-130, 2006

2. Edwards BK, Brown ML, Wingo PA, Howe HL, Ward E, Ries LA, et al: Annual report to the nation on the status of cancer, 1975-2002, featuring population-based trends in cancer treatment. J Natl Cancer Inst 97: 1407-1427, 2005.

3. Bromberg JF: Activation of STAT proteins and growth control. Bioessays 23: 161-169, 2001.

4. Buettner R, Mora LB and Jove R: Activated STAT signaling in human tumors provides novel molecular targets for therapeutic intervention. Clin Cancer Res 8: 945-954, 2002.

5. Catlett-Falcone R, Landowski TH, Oshiro MM, Turkson J, Levitzki A, Savino R, Ciliberto G, Moscinski L, FernandezLuna JL, Nunez G, Dalton WS and Jove R: Constitutive activation of Stat 3 signaling confers resistance to apoptosis in human U266 myeloma cells. Immunity 10: 105-115, 1999.

6. Gao B, Shen X, Kunos G, Meng Q, Goldberg ID, Rosen EM and Fan S: Constitutive activation of JAK-STAT3 signaling by BRCA1 in human prostate cancer cells. FEBS Lett 488: 179-184, 2001

7. Al Zaid Siddiquee K and Turkson J: STAT3 as a target for inducing apoptosis in solid and hematological tumors. Cell Res 18: 254-267, 2008.

8. Lo HW, Hsu SC, Ali-Seyed M, Gunduz M, Xia W, Wei Y, Bartholomeusz G, Shih JY and Hung MC: Nuclear interaction of EGFR and STAT3 in the activation of the iNOS/NO pathway. Cancer Cell 7: 575-589, 2005.

9. Park OK, Schaefer TS and Nathans D: In vitro activation of Stat3 by epidermal growth factor receptor kinase. Proc Natl Acad Sci USA 93: 13704-13708, 1996.

10. Turkson J and Jove R: STAT proteins: novel molecular targets for cancer drug discovery. Oncogene 19: 6613-6626, 2000.

11. Bromberg J and Darnell JE Jr: The role of STATs in transcriptional control and their impact on cellular function. Oncogene 19: 2648-2673, 2000.

12. Landen CN Jr, Lin YG, Armaiz Pena GN, et al: Neuroendocrine modulation of signal transducer and activator of transcription 3 in ovarian cancer. Cancer Res 67: 10389-10396, 2007.

13. Duan Z, Foster R, Bell DA, Mahoney J, Wolak K, Vaidya A, Hampel C, Lee $\mathrm{H}$ and Seiden MV: Signal transducers and activators of transcription 3 pathway activation in drug-resistant ovarian cancer. Clin Cancer Res 12: 5055-5063, 2006.

14. Nefedova Y and Gabrilovich DI: Targeting of Jak/STAT pathway in antigen presenting cells in cancer. Curr Cancer Drug Targets 7: 71-77, 2007.

15. Schlessinger K and Levy DE: Malignant transformation but not normal cell growth depends on signal transducer and activator of transcription 3. Cancer Res 65: 5828-5834, 2005.

16. Cai L, Zhang G, Tong X, You Q, An Y, Wang Y, Guo L, Wang T, Zhu D and Zheng J: Growth inhibition of human ovarian cancer cells by blocking STAT3 activation with small interfering RNA. Eur J Obstet Gynecol Reprod Biol 148: 73-80, 2010.
17. Yang L, Ma X, Xiao L, Tang M, Weng X, Sun L and Cao Y: Uniquely modified RNA oligonucleotides targeting STAT3 suppress melanoma growth both in vitro and in vivo. Cancer Biol Ther 8: 2065-2072, 2009.

18. Gu J, Li G, Sun T, Su Y, Zhang X, Shen J, Tian Z and Zhang J: Blockage of the STAT3 signaling pathway with a decoy oligonucleotide suppresses growth of human malignant glioma cells. J Neurooncol 89: 9-17, 2008.

19. Souissi I, Najjar I, Ah-Koon L, et al: A STAT3-decoy oligonucleotide induces cell death in a human colorectal carcinoma cell line by blocking nuclear transfer of STAT3 and STAT3-bound NF-кB. BMC Cell Biology 12: 14, 2011.

20. Zhang X, Liu P, Zhang B, Wang A and Yang M: Role of STAT3 decoy oligodeoxynucleotides on cell invasion and chemosensitivity in human epithelial ovarian cancer cells. Cancer Genet Cytogenet 197: 46-53, 2010.

21. Leong PL, Andrews GA, Johnson DE, Dyer KF, Xi S, Mai JC, Robbins PD, Gadiparthi S, Burke NA, Watkins SF and Grandis JR: Targeted inhibition of Stat 3 with a decoy oligonucleotide abrogates head and neck cancer cell growth. Proc Natl Acad Sci USA 100: 4138-4143, 2003.

22. Zhang X, Zhang L, Wang L, Wei H and Tian Z: Therapeutic effects of STAT3-decoy oligodeoxynucleotide on human lung cancer in xenograft mice. BMC Cancer 7: 149, 2007.

23. Devalapally H, Duan Z, Seiden MV and Amiji MM: Modulation of drug resistance in ovarian adenocarcinoma by enhancing intracellular ceramide using tamoxifen-loaded biodegradable polymeric nanoparticles. Clin Cancer Res 14: 3193-3203, 2008.

24. Mao HL, Liu PS, Zheng JF, Zhang PH, Zhou LG, Xin G and Liu C: Transfection of Smac/DIABLO sensitizes drug-resistant tumor cells to TRAIL or paclitaxel-induced apoptosis in vitro. Pharmacol Res 56: 483-492, 2007.

25. Bromberg J: Stat proteins and oncogenesis: J Clin Invest 109: 1139-1142, 2002.

26. Fletcher S, Turkson J and Gunning PT: Molecular approaches towards the inhibition of the signal transducer and activator of transcription 3 (Stat3) protein. ChemMedChem 3: 1159-1168, 2008.

27. Leeman RJ, Lui VW and Grandis JR: STAT3 as a therapeutic target in head and neck cancer. Expert Opin Biol Ther 6: 231-241, 2006.

28. Colomiere M, Findlay J, Ackland L and Ahmed N: Epidermal growth factor-induced ovarian carcinoma cell migration is associated with JAK2/STAT3 signals and changes in the abundance and localization of alpha6betal integrin. Int $\mathrm{J}$ Biochem Cell Biol 41: 1034-1045, 2009.

29. Jing $N$ and Tweardy DJ: Targeting Stat3 in cancer therapy. Anticancer Drugs 16: 601-607, 2005.

30. Shen J, Li R and Li G: Inhibitory effects of decoy-ODN targeting activated STAT3 on human glioma growth in vivo. In Vivo 23: 237-243, 2009.

31. Liotta LA and Stetler-Stevenson WG : Tumor invasion and metastasis: an imbalance of positive and negative regulation. Cancer Res 51 (Suppl 18): 5054S-5059S, 1991.

32. Belotti D, Paganoni P, Manenti L, Garofalo A, Marchini S, Taraboletti G and Giavazzi R. Matrix metalloproteinases (MMP9 and MMP2) induce the release of vascular endothelial growth factor (VEGF) by ovarian carcinoma cells: implications for ascites formation. Cancer Res 63: 5224-5229, 2003.

33. dos Santos LG, Lopes-Costa PV, dos Santos AR, Facina G and da Silva BB: Bcl-2 oncogene expression in estrogen receptorpositive and negative breast carcinoma. Eur J Gynaecol Oncol 29: 459-461, 2008.

34. Alas S and Bonavida B: Inhibition of constitutive STAT3 activity sensitizes resistant non-Hodgkin's lymphoma and multiple myeloma to chemotherapeutic drug-mediated apoptosis. Clin Cancer Res 9: 316-326, 2003.

35. Selvendiran K, Koga H, Ueno T, et al: Luteolin promotes degradation in signal transducer and activator of transcription 3 in human hepatoma cells: an implication for the antitumor potential of flavonoids. Cancer Res 66: 4826-4834, 2006.

36. Enari M, Sakahira H, Yokoyama H, Okawa K, Iwamatsu A and Nagata S: A caspase-activated DNase that degrades DNA during apoptosis and its inhibitor ICAD. Nature 391: 43-50, 1998.

37. Kamada S, Kikkawa U, Tsujimoto Y and Hunter T: Nuclear translocation of caspase- 3 is dependent on its proteolytic activation and recognition of a substrate-like protein(s). J Biol Chem 280: $857-860,2005$. 